Published by Al-Nahrain College of Medicine P-ISSN 1681-6579

E-ISSN 2224-4719

Email: iraqijms@colmed-alnahrain.edu.iq http://www.colmed-alnahrain.edu.iq http://www.iraqijms.net Iraqi JMS 2018; Vol. 16(1)

\title{
Combining Strain Elastography Findings with Ultrasound BIRADS System to Discriminate between Benign and Malignant Solid Breast Masses
}

\author{
Raad H.A. Tawfeeq ${ }^{1} D M R D, F I B M S-R D$, Enam A. Khalel ${ }^{2} D M R D, F R C P$, Noor K.N. Al-Waely ${ }^{3}$ FIBMS- \\ $R D$
}

${ }^{1}$ Acting Chairman of the Iraqi Board of Radio-Diagnosis, ${ }^{2} \mathrm{Head}$ of radiology department in Oncology Teaching Hospital, Baghdad Medical City, Iraq, ${ }^{3}$ Dept. of Surgery, College of Medicine, Al-Nahrain University, Baghdad, Iraq

\begin{abstract}
Background Elastography has been attracting attention as a new non-invasive diagnostic tool with the potential to improve breast masses characterization.

Objective

To assess the value of incorporating strain elastography into the ultrasound Breast Imaging Reporting and Data System (BIRADS) System to differentiate benign from malignant breast masses.

Methods Fifty-six women with 61 solid breast masses were enrolled in this study. Ultrasound was performed and the mass was given an US BIRADS category. Elastographic examination was performed and each lesion was assigned an Elasticity Score (ES) according to the Tsukuba scoring system. Strain Ratios (SRs) were calculated from a tumor adjusted Region of Interest (ROI) and a reference ROI in the fatty tissue. The US BIRADS was modified according to the elasticity criteria. Sensitivity, specificity, area under the curve (AUC) and cutoff values were calculated for US BIRADS, ES, SR and the modified BIRADS method using (ROC) curve analysis.

Results The final results were based on 61 masses, 25 benign and 36 malignant. The sensitivity and specificity were respectively ( $97 \%$ and $80 \%)$ for US BIRADS, $(86.1 \%$ and $84 \%)$ for ES, $(94.44 \%$ and $84 \%)$ for SR, and (97\% and $84 \%$ ) for the modified BIRADS.

Conclusion Combining elastography with conventional ultrasound yielded better diagnostic performance with improved specificity.

Keywords Elastography, breast masses, elasticity, strain ratio, modified BIRADS

Citation Tawfeeq RHA, Khalel EA, Al-Waely NKN. Combining strain elastography findings with ultrasound BIRADS system to discriminate between benign and malignant solid breast masses. Iraqi JMS. 2018; Vol. 16(1): 74-83. doi: 10.22578/IJMS.16.1.11
\end{abstract}

List of abbreviations: $A C R=$ American College of Radiology, AUC $=$ Area under curve, $B$-mode $=$ Brightness mode, $B I R A D S=$ Breast imaging reporting and data system, ES = Elasticity score, FNAC = Fine needle aspiration cytology, FOV $=$ Field of view, ROC $=$ Receiver operating characteristic, $\mathrm{ROI}=$ Region of interest, $\mathrm{SR}=$ Strain ratio, $\mathrm{US}=$ Ultrasound

\section{Introduction}

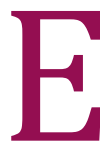
lastography is a recently introduced ultrasound technology (1) with the potential to visually and objectively assess the elastic properties of tissues previously assessed roughly by physical palpation (2-4).

It has the potential to differentiate benign from malignant lesions (5) as cancerous tissue becomes harder due to cell proliferation and angiogenesis (1). Two main elastographic technologies used in clinical practice are strain elastography and shear wave imaging which differ by the method utilized to displace the 
tissue and the imaging system, which quantify the magnitude of displacement and converts it into a color-coded map termed elastogram ${ }^{(6)}$. One of the earliest applications of elastgraphy in medical field is in breast lesion characterization. It is considered a useful adjunct to the standard $B$ mode imaging. Initial clinical trials showed that strain elastography has the potential to improve breast masses characterization ${ }^{(6)}$.

The method most widely used for classifying the elastographic images is the Tsukuba scoring system proposed by Dr. Ueno et al ${ }^{(7-9)}$, which uses a five-point scale to classify elastograms. This system has been shown to have a diagnostic performance comparable to the US BIRADS system in evaluating breast lesions for malignant properties ${ }^{(10,11)}$.

Elastography has been added to the ACR ultrasound BIRADS lexicon $2013^{(12)}$ but its exact role has not been defined. Because elastography cannot be used in isolation for breast lesion evaluation and other ultrasound parameters should be taken into consideration, several methods were proposed to combine the elastographic criteria of breast masses with the US BIRADS to better assess the lesion for malignant potential, thereby decreasing the false negative biopsies ${ }^{(13-15)}$. An Italian study has used only elastographic parameters to elucidate a scaling system for breast lesion characterization (16); however, only few studies incorporated both elasticity score and strain ratio measurement to modify the US BIRADS $(17,18)$.

This work was done to evaluate the clinical usefulness of a proposed method of modifying the B-mode dependent US BIRADS making use of the elastographic parameters of breast lesions namely: elasticity scoring (ES) and strain ratio (SR).

\section{Methods}

\section{Patients and Data Collection}

This analytic cross-sectional study was conducted in the breast clinic at the Oncology Teaching Hospital, Baghdad, Iraq from April 2014 to April 2015, a period during which, 62 patients with 68 solid breast masses were examined.

The patients included in the study were the females presented for the first time with solid breast mass visible on B-mode ultrasound that is either palpable or incidentally discovered by other imaging modalities. Follow up was obtained for some masses (No. $=15$ ) with benign ultrasound and elasticity features for whom FNAC was done as the pathological diagnosis (pathological analysis detailed later).

Excluded from the study patients with previous surgical intervention, patients with BIRADS 0 masses, patients who received chemotherapy and patients with benign looking masses who refused FNAC in the first place.

Out of all patients who were examined (No. =62), 6 patients were excluded as no follow up data were obtained. Thus, the final data analysis was based on 61 solid breast masses obtained from 56 women. Informed verbal consent was obtained from each patient.

\section{Examination Technique}

Conventional B-mode ultrasound was performed with the patient in the supine position then elastographic examination was done using high end ultrasound system (GE healthcare, Voluson E6) with high frequency linear probe (10-14 MHz). For elastographic examination, the field of view (FOV) was set so that the lesion is not at the periphery and does not exceed $1 / 2$ of the FOV with the inclusion of adequate surrounding normal breast tissue, subcutaneous fat and the pectoral muscles where feasible.

The elastographic examination was accomplished by applying very light touch with the transducer perpendicular to the skin and to the lesion, a minimum of 5-6 compression release cycles were applied trying not to have lateral movement.

\section{Measurements and Image Interpretation}

On B-mode examination the location of each lesion was labeled including the side, the O'clock face, the distance from the nipple and the depth of the lesion. The lesion was assigned an ultrasound BIRADS category by the joint 
decision of 2 radiologists. The maximum dimension of the lesion in the B-mode image was measured. Each lesion was assigned an elasticity score according to the Tsukuba elasticity score system. Measuring the strain ratio was achieved by placing 2 circles of approximately equal diameter, one in the subcutaneous fat adjacent to the lesion (reference) and the other in the lesion (ROI) and the diameter was calibrated so that to include the lesion without extending into the adjacent tissues.

\section{Elastography Based BIRADS Modification}

Our proposed system for modifying US BIRADS (figure 1) was applied to BIRADS 3 and BIRADS 4 lesions based on the cut off points for elasticity score $(>3)$ and strain ratio $(>3.2)$ derived from the ROC curve analysis.

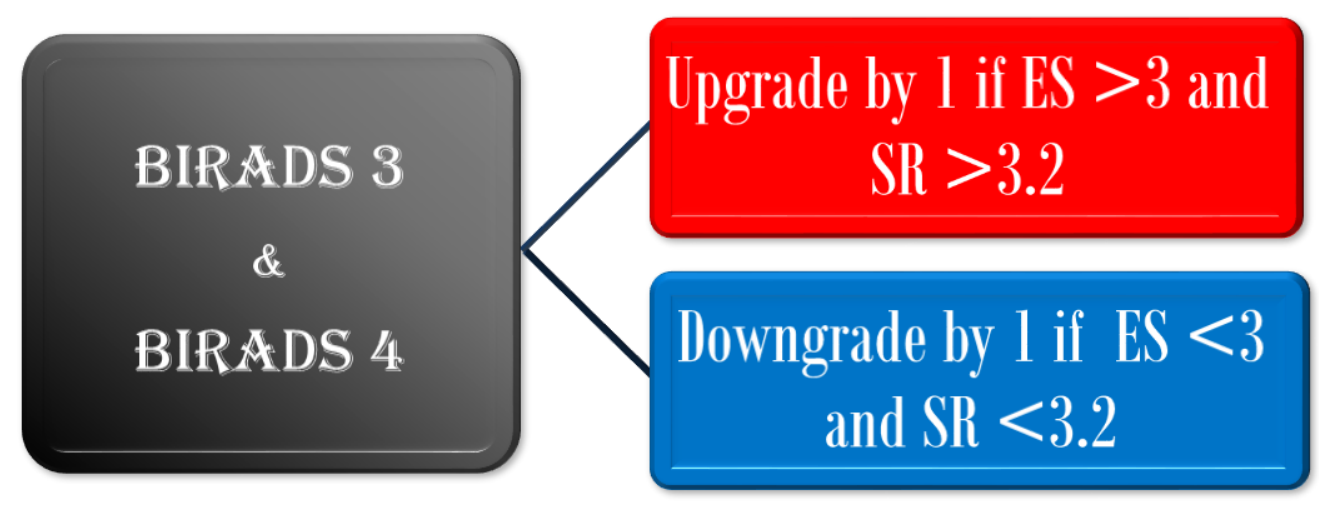

Figure 1. A schematic drawing illustrating the BIRADS modification method

\section{Pathological Analysis}

Our final diagnosis was based on pathological analysis of breast mass samples obtained with fine needle aspiration cytology (FNAC) (No. $=15$ ), needle biopsy (No. $=10$ ), excision biopsy (No. $=16)$, or radical surgery (No. $=20$ )

FNAC was considered the standard only for masses with benign ultrasonographic and elastographic appearances with concordant FNAC results, for those patients 3 months follow up with ultrasound was performed which showed no interval change in size.

For lesions with discordant ultrasonographic and elastographic diagnoses or for whom the FNAC results were suspicious biopsy was performed to confirm the diagnosis with the exception of 3 BIRADS 3 lesions, which had elasticity score suggestive of malignancy (score 4) but they had calcification with benign ultrasound features consistent with calcified fibroadenoma, in addition, in 2 of these masses mammograms were available which confirmed internal calcification, so we depended on the FNAC results plus follow up and no biopsy was performed. A net of 25 benign and 36 malignant breast masses were enrolled in the study.

\section{Statistical Analysis}

Data collection and descriptive statistics including the graph design were accomplished via Microsoft excel 2010. Cross tables were built using Statistical Package for Social Sciences (SPSS) statistics for windows, version 22.0.

Comparing the mean SR for benign and malignant masses was performed using independent student test (t-test).

The diagnostic performance of the parameters incorporated in the study was assessed by using receiver operating characteristic (ROC) curve analysis based on De Long et al method with calculation of the sensitivity and specificity in addition to cut off point calculation based on Youden index. The same De Long method was used for pairwise comparison between different 
ROC curves. The above mentioned statistical tests (t-test, ROC curve analysis, ROC curves comparison) were performed using MedCalc for Windows, version 14.12.0 (MedCalc Software, Ostend, Belgium). For all tests, a $\mathrm{P}$ value of less than 0.05 was considered to indicate a statistically significant difference.

\section{Results}

The patients' population consisted of 56 women with 61 solid breast masses, mean age of the patients was 44.7 years; age range $20-65$ years. Of these women, 42 presented with palpable breast mass while 14 women had their masses discovered incidentally on imaging performed for other indications. The mean diameter for the masses was $1.65 \mathrm{~cm}$, range $0.4-3 \mathrm{~cm}$, mean diameter for benign masses was $1.47 \mathrm{~cm}$, and mean diameter for malignant masses was 1.79 $\mathrm{cm}$.

\section{Ultrasound BIRADS}

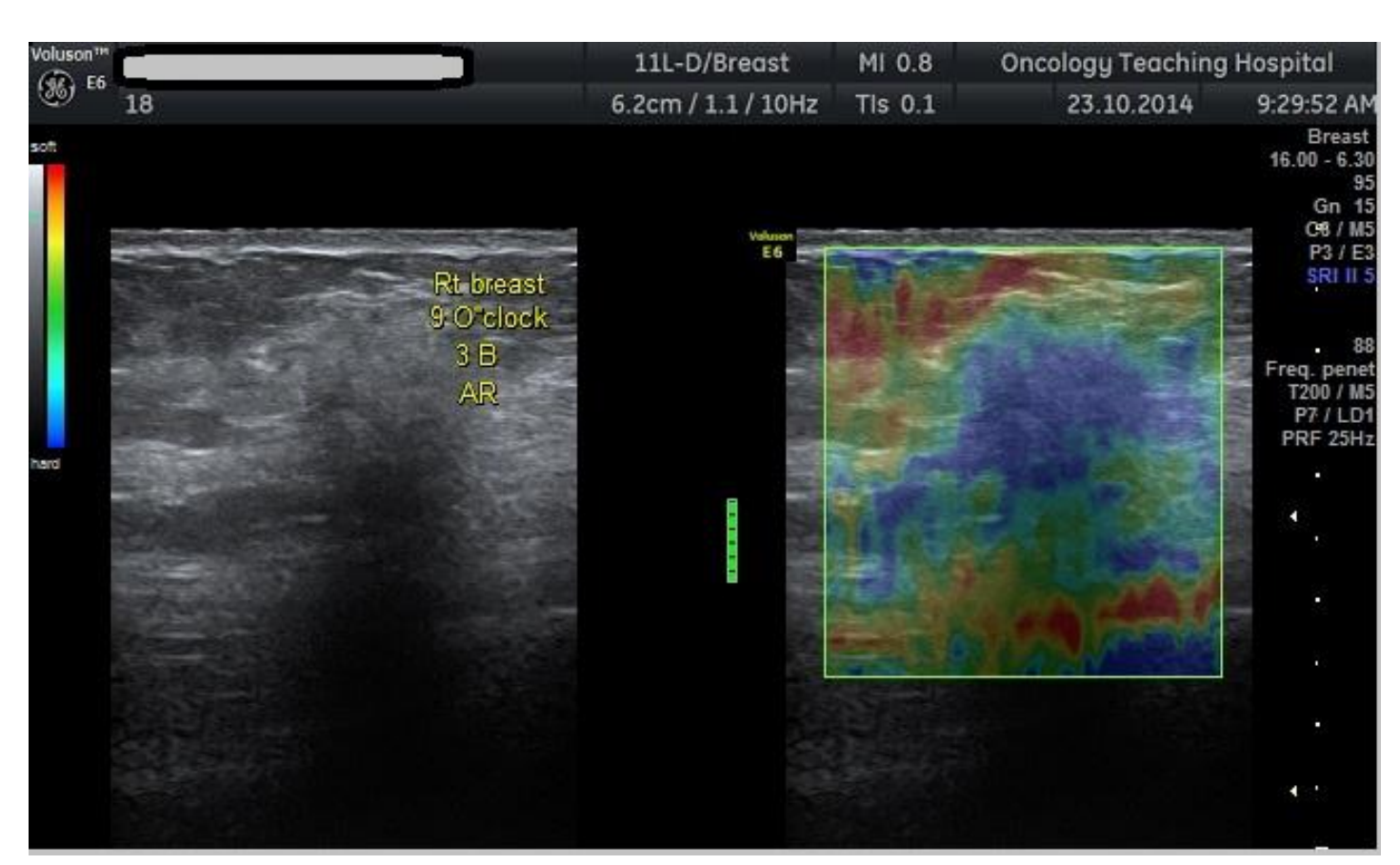

Figure 2. Malignant mass with elasticity score of $\mathbf{5}$. The tumor margins are indistinct on B-mode; on the contrary elastogram better depicts the tumor margins

Twenty-one of the masses included in the study were classified as BIRADS 3, 26 as BIRADS 4 and 14 as BIRADS 5. Of the masses given BIRADS 5 category, all were malignant; BIRADS 3 masses had one mass, which proved to be malignant, and 21 masses given BIRADS 4 were malignant. The diagnostic performance of the US BIRADS was evaluated by ROC curve analysis; the area under the curve (AUC) was 0.925. US BIRADS had sensitivity and specificity of $97 \%$ and $80 \%$ respectively considering scores 4 and 5 malignant and scores 1, 2, and 3 benign.

\section{Elasticity Score}

Out of the four masses given an ES of 1, none was malignant. On the other hand, $87.5 \%$ (14 out of 16) of score 2 masses that were benign. Six masses were given an ES of 3 ( 3 were benign and 3 malignant), $80 \%$ (16 out of 20 ) of masses given Tsukuba score 4 were malignant while all fifteen masses which were given a score of 5 were malignant (figures 2). 
Of the masses given an elasticity score 4, 20\% (4 out of 20) were benign. likewise, $12.5 \%$ ( 2 out of 16) of masses with a score of 2 were malignant. The overall diagnostic performance of elasticity score was evaluated using (ROC) curve; with a cutoff point (> 3) ES had a sensitivity and specificity of $86.1 \%$ and $84 \%$ respectively.

\section{Strain Ratio}

The distribution of masses according to SR is shown in figure 3 . Thirteen out of twenty-five $(52 \%)$ of benign masses with strain ratio less than 2.1. On the other hand, 19 out of 36 (52.7\%) of malignant masses had strain ratios $\geq$ 5.1 (Figure 4).

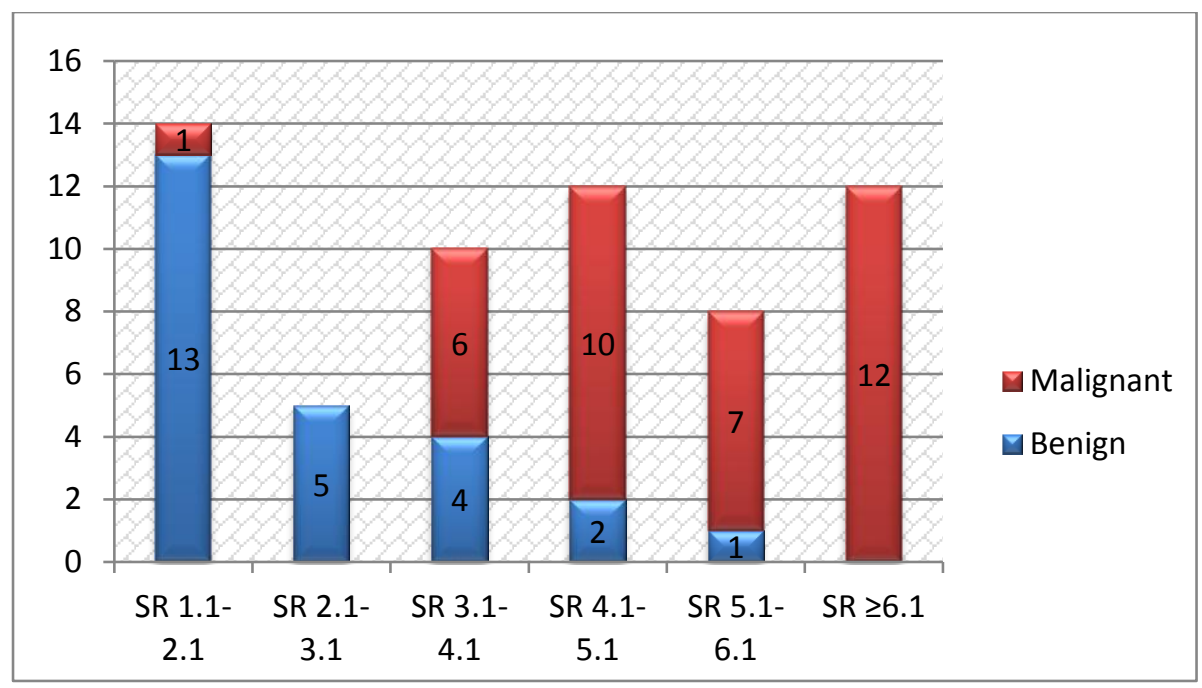

Figure 3. Distribution of strain ratios in benign and malignant masses

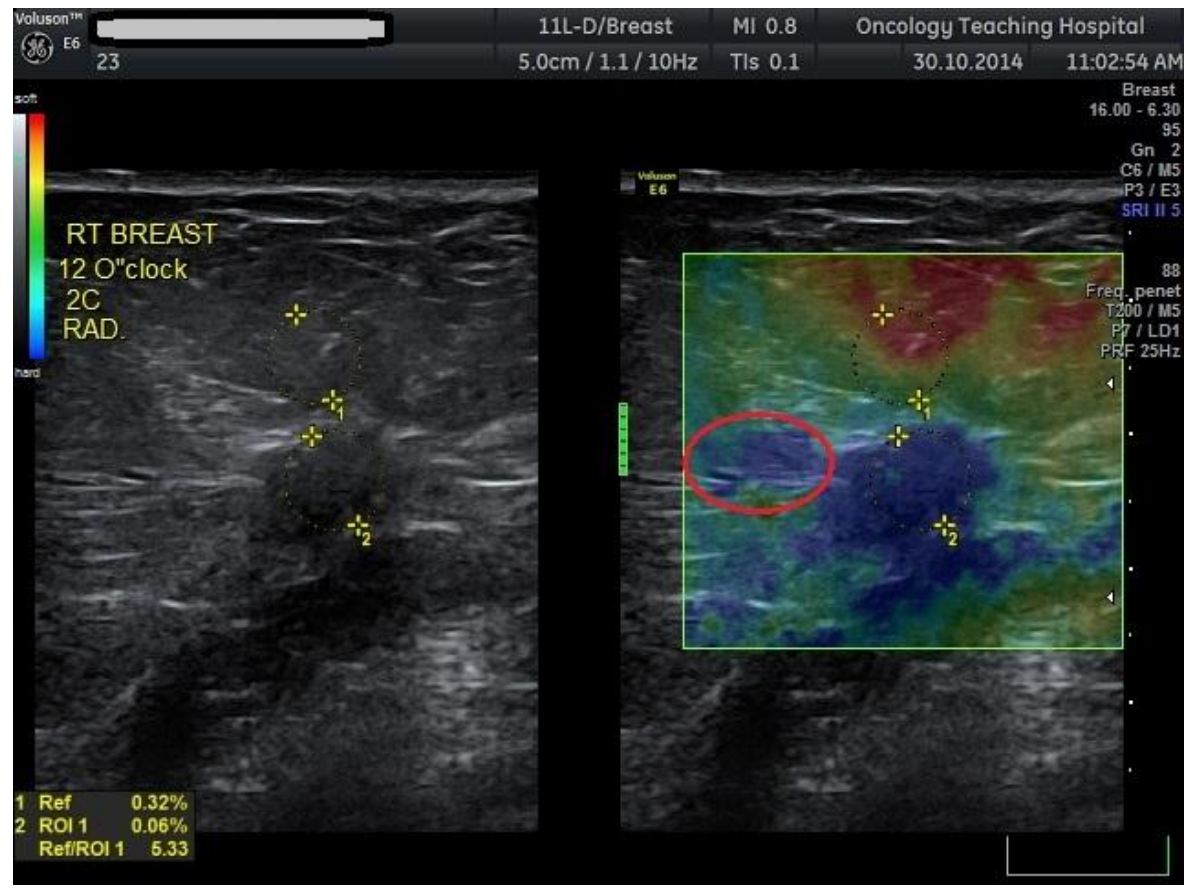

Figure 4. Malignant mass with ES 4 and SR of 5.33. There is a band of stiffness (circle) extending from the main tumor not well depicted at B-mode which could possibly represents intraductal extension 
The mean strain ratio for all masses included in the study was 4.3, mean SR for benign masses 2.4, mean SR for malignant masses was 5.6. Independent sample t-test was used to evaluate whether the difference between the mean SR for benign and malignant masses was statistically significant. The result of the test showed significant difference between the two means ( $P$ value $<0.0001$ )

The overall diagnostic performance of Strain Ratio measurement was evaluated with ROC curve, AUC was 0.936 with a cut off value (>3.2), SR had sensitivity and specificity of $94.44 \%$ and $84 \%$ respectively. Using comparison between ROC curves, there was no significant difference between the diagnostic performance of ES and SR ( $P$ value $=0.56$ ) .

Comparing the diagnostic performance of ES and SR with the ultrasound BIRADS showed no statistically significant difference with a $P$ value of 0.8299 and 0.7990 respectively.

Moreover, no statistically significant difference between diagnostic performance of ES and SR.

\section{Assessing the Proposed Modified BIRADS}

Thirteen masses categorized as BIRADS 3 have been downgraded to BIRADS 2 and were benign. Likewise, four masses categorized as BIRADS 4 have been downgraded into BIRADS 3 and proved to be benign (Table 1 ).

Table 1. Modified BIRADS vs. BIRADS

\begin{tabular}{ccccccc}
\hline \multirow{2}{*}{ Mass } & \multirow{2}{*}{ BIRADS } & \multicolumn{4}{c}{ Modified } \\
& & Category 2 & Category 3 & Category 4 & Category 5 & Total \\
\hline \multirow{2}{*}{ Benign } & Category 3 & $13^{*}$ & 4 & $3^{*}$ & & 20 \\
& Category 4 & 0 & $4^{*}$ & 1 & & 5 \\
\multirow{2}{*}{ Malignant } & Category 3 & & 1 & 0 & 0 & 1 \\
& Category 4 & & 0 & 1 & $20^{*}$ & 21 \\
\hline
\end{tabular}

On the other hand, three BIRADS category 3 masses have been upgraded into BIRADS 4 and they were benign. Eighteen BIRADS category 4 masses have been upgraded to BIRADS 5 and proved malignant.

The diagnostic performance of the modified BIRADS was excellent as assessed by the ROC curve analysis with sensitivity $97 \%$, specificity $84 \%$ and $A U C=0.984$ when a cut-off point of $>3$ was considered.

Comparing the diagnostic performance of the modified BIRADS with the other diagnostic parameters used in this study showed significant difference between the modified BIRADS and standard BIRADS $(P=0.0251)$ and between modified BIRADS and ES ( $P=0.0068)$. On the other hand, no statistically significant difference was found between the modified BIRADS and SR diagnostic performance $(P=0.0884)$.

\section{Discussion}

This study showed good elastography diagnostic performance, comparable to the US BIRADS, the currently widespread system for breast masses characterization. Elastography, although showed lower sensitivity than BIRADS in the study, had higher specificity with the potential to reduce the rate of negative biopsies. The proposed method for integrating elastography in the US BIRADS system showed better overall diagnostic performance as compared to US BIRADS alone.

\section{Elasticity Score}

Tsukuba elasticity scoring is useful for differentiating benign from malignant breast lesions. Two of the 5 false negative masses had ES of 2 and 3 had an ES of 3. Three masses had a maximum diameter exceeding $2 \mathrm{~cm}$ and one was deeply located within the breast, which 
could reflect the difficulty in obtaining good elastographic images in large and in deeply located masses. This is in concordance with the study by Ciurea et al., (19) which showed difficulty in obtaining good quality elastographic images in large and in deep masses near the chest wall. There has been difficulty in categorizing masses with mixed strain color pattern (blue and green) as Tsukuba score 2 masses are defined as masses in which, there is mixed green and blue colors with no reference as to the predominant color pattern. Tsukuba score 4 pattern as described originally should have no strain over the whole lesion meaning the lesion appears blue all over. In this work we encountered three lesions with mixed color pattern but predominance of blue color was noted, we have given them as score 2 . Two of these masses were malignant and one was fibroadenoma with calcification.

Of the 20 masses which had an ES 4, four were benign. These masses had calcification, which could account for the lack of strain in these lesions. In our work, we noticed better definition of lesion margins at elastography for some malignant masses which had indistinct margins with acoustic shadowing (Figure 2). Also, in some cases a band of stiffness was noted extending from the lesion in a course likened to the ductal anatomy which could represent intraductal extension (Figure 4). The results for Tsukuba elasticity score is consistent with the original work done by Itoh et al. ${ }^{(7)}$ who first described this scoring system. They found a sensitivity of $86.5 \%$ and a specificity of $89.8 \%$, values which are comparable to ours, using the same cut off value (>3) for benign versus malignant lesions differentiation. In their study all lesions with elasticity score of 1 were benign. Several other studies ${ }^{(14,20-23)}$ showed improved specificity for elasticity scoring compared to the US BIRADS.

\section{Strain Ratio}

Strain ratio measurement showed better diagnostic performance than ES with a sensitivity of $94.4 \%$ and specificity of $84 \%$. Two masses had false negative results. One mass had SR of 1.9 far less than our calculated cut off point of 3.2. This mass had a maximum dimension of $2.5 \mathrm{~cm}$, which could affect the quality of the elastogram obtained. The other false negative mass had a SR of 3.2 (our cut off value). It was located deep within the breast which can potentially affect the quality of elastogram obtained. Four benign masses had SR above our cut off value. Two of them were calcified and one had a maximum dimension of 2.3. The fourth mass was located in a predominantly fatty breast which could account for the relatively high strain ratio as compared to fat.

Zhi et al. (24), in concordance with our results, concluded that strain ratio provides a more reliable diagnostic performance in comparison to Tsukuba scoring system for elastography with sensitivity and specificity $92.4 \%$ and $91.1 \%$ respectively with a cutoff point of 3.05 .

Farrok et al. ${ }^{(22)}$ concluded that strain ratio would help increase the specificity of elastography. In their study, they did not report the cut off value for discriminating benign from malignant masses.

\section{Proposed Modified BIRADS}

In our proposed system for BIRADS modification we used elasticity score and strain ratio criteria to re-categorize BIRADS 3 and 4 masses. Our method for modifying the US BIRADS according to the elasticity score and strain ratio measurements showed improved specificity as compared to the US BIRADS alone ( $84 \%$ vs. $80 \%$ ) with no reduction in sensitivity $(97 \%)$. In the current study, 18 out of 21 masses with BIRADS 3 category were downgraded to BIRADS 2 and all proved benign (Figure 5) our masses classified as BIRADS 4 were downgraded into BIRADS 3 and all were benign. On the other hand, three benign masses originally categorized as BIRADS 3 were upgraded into BIRADS 4 which is a drawback for our modified system. BIRADS 4 masses, which has been upgraded into BIRADS 5 (No. =18), all proved malignant. Albeit the increase in specificity in 
the modified system is not huge, the clinical implications would be of paramount importance. Downgrading BIRADS 3 lesion to BIRADS 2 means less patient anxiety. In addition, no further follow up is needed decreasing the cost burden to the patient and to the health institutions. In a similar fashion downgrading benign BIRADS 4 masses into BIRADS 3 would be even more beneficial as this would spare the patient the unnecessary biopsy reflecting the core value of introducing elastography in breast imaging algorithm.
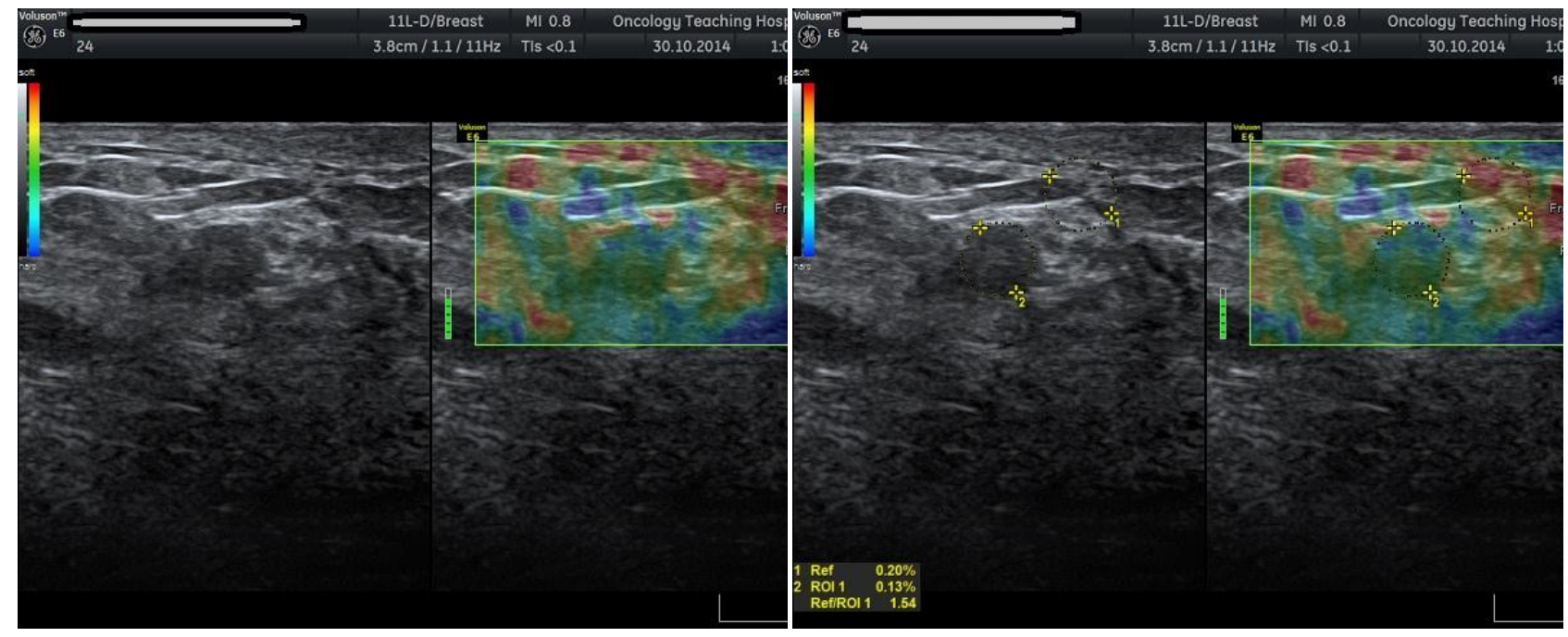

Figure 5. BIRADS 3 mass downgraded to BIRADS $2(E S=1, S R=1.54)$

\section{Limitations}

One of the limitation encountered in this study is the overrepresentation of malignant lesions, which is actually related to the fact that our cases were examined in the Breast Clinic at the Oncology Teaching Hospital, which is considered a tertiary center so we are, by virtue of the place, more likely to encounter malignant masses than in a usual everyday practice. Another limitation is the fact that not all masses were confirmed histopathologically as we were obliged to follow the policies and procedures adopted at the place we were working at which, state that a mass with benign findings at ultrasound would be followed to confirm its benign nature with no role for invasive measures in such masses.

The current study results concluded that using strain elastography, there is significant difference between benign and malignant solid breast masses, however; overlap still exists. Strain ratio measurement had better diagnostic performance than elasticity score. By incorporating elasticity assessment in the US BIRADS categorization increased specificity was noted which may reduce the rate of unnecessary biopsy. Applying our modified BIRADS system would also change the clinical course of BIRADS 3 lesions with profound clinical impacts both on the patient and the health institutions and potential reduction in the number of BIRADS 3 lesions requiring follow up.

\section{Acknowledgments}

The authors are extremely indebted to all women who willingly participated in this work. Special thanks to Dr. Hamad Shihab Hamad, senior radiologist at Al-Shaheed Ghazi Al-Harriri Hospital for his review of the main manuscript.

\section{Authors Contribution}

All authors contributed equally to the design of the study and data analysis. Data collection, statistical analysis and writing the manuscript 
was done by Dr. Nee'ma. Dr. Tawfeeq and Dr. Khalel have thoroughly reviewed the article and contributed to the writing of the final manuscript.

\section{Conflict of interest}

The authors declare no conflict of interest.

\section{Funding}

None.

\section{References}

1. Nakashima K, Shiina T, Sakurai $M$, et al. JSUM ultrasound elastography practice guidelines: breast. J Med Ultrason. 2013; 40(4): 359-91. doi: 10.1007/s10396-013-0457-0.

2. Ophir J, Céspedes I, Ponnekanti H, et al. Elastography: a quantitative method for imaging the elasticity of biological tissues. Ultrason Imaging. 1991; 13(2): 11134. doi: 10.1177/016173469101300201.

3. Samani A, J. Zubovits, Plewes D. Elastic moduli of normal and pathological human breast tissues: an inversion-technique-based investigation of 169 samples. Phys Med Biol. 2007; 52(6): 1565-76. doi: 10.1088/0031-9155/52/6/002.

4. Frey $H$. Realtime elastography. [A new ultrasound procedure for the reconstruction of tissue elasticity]. Radiologe. 2003; 43: 850-5. doi: 10.1007/s00117-0030943-2.

5. Barr RG. Sono-Elastography: main clinical applications. 1st ed. EDIMES, 2014. p. 49-68.

6. SonoWorld. Elastography: What are the clinical applications for elastography? [cited 2017 April 15]. URL:

https://sonoworld.com/Client/MiniSites/MiniSiteDet ails.aspx?MiniSiteld=1

7. Itoh A, Ueno E, Tohno E, et al. Breast disease: clinical application of US elastography for diagnosis. Radiology. 2006; 239(2): 341-50. doi: 10.1148/radiol.2391041676.

8. Tardivon A. Real-time elasticity helps to improve specificity. Eur Radiol. 2009; 19: 1621-8.

9. Ueno $E$, Itoh $A$. Diagnosis of breast cancer by elasticity imaging. Eizo Joho Medical. 2004; 36: 2-6.

10. Duma M, Chiorean A, Dudea S, et al. Breast lesions: correlations between ultrasound BI-RADS classification and UENO-ITOH elastography score. Ultraschall Med 2008; 29 - OP_2_12. doi: 10.1055/s2008-1079811.

11. Chiorean A, Duma MM, Dudea S, et al. Short analysis on elastographic images of benign and malignant breast lesions based on color and hue parameters. Ultraschall Med. 2008; 29 - OP_2_13. doi: 10.1055/s2008-1079812.
12. Mendelson EB, Böhm-Vélez $M$, Berg WA, et al. ACR BIRADS $^{\circledR}$ Ultrasound. In: ACR BI-RADS ${ }^{\circledR}$ Atlas, breast imaging reporting and data system. Reston, VA, American College of Radiology; 2013.

13. Zhi H, Ou B, Luo BM, et al. Comparison of ultrasound elastography, mammography, and sonography in the diagnosis of solid breast lesions. J Ultrasound Med. 2007; 26(6): 807-15.

14. Lee JH, Kim SH, Kang BJ, et al. Role and clinical usefulness of elastography in small breast masses. Acad Radiol. 2011; 18(1): 74-80. doi: 10.1016/j.acra.2010.07.014.

15. Zhi $H$, Xiao $X Y, O u B$, et al. Could ultrasonic elastography help the diagnosis of small $(\leq 2 \mathrm{~cm})$ breast cancer with the usage of sonographic BI-RADS classification? Eur J Radiol. 2012; 81(11): 3216-21. doi: 10.1016/j.ejrad.2012.04.016.

16. Maggini E, Mancuso E, Medvedyeva $O$, et al. Quantitative elastosonography in the diagnosis of breast nodules: assessment of a multiparametric analysis. ECR 2014Type: Scientific Exhibit. Poster No.: C-0394 Congress. doi: 10.1594/ecr2014/C-0394.

17. Moukhtar FZ, Abu EL Maati AA. Real-time tissue elastography combined with BIRADS-US classification system for improving breast lesion evaluation. The Egyptian J Radiol Nuclear Med. 2014; 45(3): 1021-8. doi: http://dx.doi.org/10.1016/j.ejrnm.2014.05.007.

18. Bojanic K, Katavic N, Smolic M, et al. Implementation of elastography score and strain ratio in combination with B-mode ultrasound avoids unnecessary biopsies of breast lesions. Ultrasound Med Biol. 2017; 43(4): 804-16. doi: 10.1016/j.ultrasmedbio.2016.11.019.

19. Ciurea Al, Dumitriu D, Ciortia C, et al. Artifacts and pitfalls in breast elastoultrasonography: pictorial assay. Med Ultrasonog J. 2008; 10(2): 93-8.

20. Yerli H, Yilmaz T, Kaskati T, et al. Qualitative and semiquantitative evaluations of solid breast lesions by sonoelastography. J Ultrasound Med. 2011; 30: 17986.

21. Fischer T, Peisker U, Fiedor $S$, et al. Significant differentiation of focal breast lesions: raw data-based calculation of strain ratio. Ultraschall in Med. 2012; 33(4): 372-9. doi: 10.1055/s-0031-1273222.

22. Farrokh A, Wojcinski S, Degenhardt F. [Diagnostic value of strain ratio measurement in the differentiation of malignant and benign breast lesions]. Ultraschall in Med. 2011; 32(4): 400-5. doi: 10.1055/s-0029-1245335.

23. Wojcinski S, Farrokh A, Weber $S$, et al. Multicenter study of ultrasound real-time tissue elastography in 779 cases for the assessment of breast lesions: improved diagnostic performance by combining the BI-RADS $^{\circledR}$-US classification system with sonoelastography. Ultraschall in Med. 2010; 31(5): 484-91. doi: 10.1055/s-0029-1245282.

24. Zhi H, Xiao XY, Yang HY, et al. Ultrasonic elastography in breast cancer diagnosis: strain ratio vs 5-point scale. 
Iraqi JMS 2018; Vol. 16(1)

Acad Radiol. 2010; 17(10): 1227-33. doi: 10.1016/j.acra.2010.05.004.

Correspondence to Dr. Noor K.N. Al-Waely
E-mail: noor.k@colmed-alnahrain.edu.iq noor83kadhem@yahoo.com

Received May $9^{\text {th }} 2017$

Accepted Sep. 24 2017 